\title{
Potential Role of ACE2 Inhibition in COVID-19 Severity
}

\author{
George Santarpia* \\ College of Public Health, University of Nebraska Medical Center, USA
}

*Corresponding author: George Santarpia, College of Public Health, University of Nebraska Medical Center, USA.

To Cite This Article: George Santarpia. Potential Role of ACE2 Inhibition in COVID-19 Severity. 2020 - 9(1). AJBSR.MS.ID.001356. DOI: 10.34297/AJBSR.2020.09.001356.

Received: 眥 May 06, 2020; Published: 眥 June 02, 2020

\section{Opinion}

In addition to being the entry point for SARS-CoV-2, ACE2 may play a role in the disease progression of severe COVID-19 cases. The five pre-existing conditions that currently lead to the highest COVID-19 mortality rates have also been linked to altered ACE2 activity and the related renin-angiotensin system. Since there are multiple ways in which SARS-CoV-2 could be inhibiting ACE2, it is very possible that ACE2 inhibition is the reason for this connection. Due to the connection between ARDS, a common cause of death in COVID-19 patients, and ACE2, it is also possible that this can lead to COVID-19 severity in some cases.

Angiotensin-converting enzyme 2 (ACE2) is a glycoprotein that converts angiotensin II (Ang II) into angiotensin 1-7 (Ang 1-7) and is an important component in the renin-angiotensin system (RAS) [1]. The RAS has many functions in the body, most of which are related to vascular function [1]. Consequently, the proper levels of ACE2 are important in maintaining cardiovascular and pulmonary health [1]. The evidence linking ACE2 to various diseases is mostly in rodent studies, as it is possible to manipulate ACE2 expression in these studies, and while there is risk of the correlations not translating to humans, the evidence is still strong. In these studies, reduced levels of ACE2 have been shown to increase atherosclerotic plaque formation, which is a main cause of heart disease [1]. Diabetes has been linked to lower levels of ACE2 and lower levels of ACE2 also increase the severity of the kidney damage related to diabetes [1]. However, there have been some studies that show that there is increased expression of ACE2 in specialized kidney cells in people with kidney disease1. This indicates that the levels of ACE2 may be important in the disease progression and the increased ACE2 expression may be protective [1]. ACE2 has also been shown to be critical in the lungs and under-expression of ACE2 in rats has been shown to increase the severity of acute respiratory distress syndrome (ARDS), while over-expression had a protective effect [1]. The RAS is essential in controlling blood pressure and the most common medicines to treat hypertension block ACE activity, which produces Ang II [1]. Therefore, it follows that ACE2 expression is also critical in maintaining blood pressure and that lower levels could cause hypertension. The data supports this, as reduced ACE2 expression was linked to spontaneously hypertensive rats and modest systolic hypertension [1]. Reduced ACE2 expression has also been correlated to the presence of cancer and cancer patients with higher ACE2 levels survived longer, on average, than patients with lower ACE2 levels [2]. This further supports the assertion that ACE2 is protective in people with these conditions. Finally, ACE2 is the cell-surface receptor for both SARS-CoV-1 and SARS-CoV-2, meaning that these viruses bind to it to enter the cell $[1,3]$.

SARS-CoV-2 is the enveloped virus responsible for the COVID-19 pandemic and has caused global concern in recent months. It causes mostly respiratory tract infections and $80 \%$ of infections are mild or asymptomatic, according to recent data4. However, it can require hospitalizations in some cases and can even result in death, which is the primary cause for concern [4]. As it only appeared a few months ago, the data on the disease is limited, but still shows definite patterns. According to data from China, the mortality rate distribution is somewhat unexpected for a few reasons. While it is not surprising that the elderly are at a higher risk of death, it is unusual that the mortality rate in children is so low. In fact, there were no deaths for those 9 and under, when the data was taken, and the mortality rate for the age range of 10-19 was actually higher, at $0.2 \%$ [4]. Usually, infants and younger children are more susceptible to disease than are older children and young adults. Also, co-morbidity is unsurprisingly a significant factor in mortality rates, but the mortality rates of specific diseases are unexpected. From most at risk to least at risk, the conditions are cardiovascular disease, diabetes, chronic respiratory disease, hypertension, and cancer [4]. Considering that COVID-19 is a respiratory infection, chronic respiratory disease is lower on the list than one would expect if the stress of the condition itself was the cause of the increased mortality rate. It is below cardiovascular disease and 
diabetes and only corresponds to a mortality rate $0.3 \%$ higher than hypertension4. Finally, the mortality rate for males is around 1.5 times higher than for females [4]. However, in China, smoking is much more common among males than it is among females and smoking can cause medical conditions that are associated with a higher mortality rate [4]. As for the cause of death, a recent study that found that $40 \%$ of hospitalized COVID-19 patients developed ARDS and -over half of them died, making ARDS responsible for many of the coronavirus deaths [4]. There have also been reports of cardiovascular complications in COVID-19 patients, including heart attacks, heart failure, and strokes, although the exact relationship between the disease and these complications is unclear [5].

Comparing the ACE2 and COVID-19 data, there are distinct connections. The diseases linked to ACE2 levels are the same diseases that increase the mortality rate the most in coronavirus patients. Also, lower levels of ACE2 have been shown to increase the severity of ARDS symptoms, which is the main cause of death for coronavirus. Finally, cardiovascular complications have been observed with this disease and the one of the main roles of ACE2 is controlling vascular function. When you consider the fact that ACE2 is the cell-surface receptor for SARS-CoV-2, it seems unlikely that this is coincidental.

Like all viruses, SARS-CoV-2 needs time to carry out its infection in the cell, which means that there will be a significant portion of infected live cells at any given time. This means that any changes in cell function the virus causes during infection could significantly impact the host. Since it binds to the ACE2 protein to enter the cell, it is possible that it is interfering with the function of that protein. One way this could happen is through receptor-destroying activity, which has been observed in animal viruses, including some coronaviruses [6]. Other possibilities include that the binding itself might also prevent ACE2 converting Ang II into Ang 1-7 by blocking the active site and that endocytosis triggered by the virus could remove ACE2 from the cell surface, where it contacts Ang II. Additionally, protein production can be limited during viral infection, as is the case with SARS-CoV-1 infections, which could further compound this problem [7]. Healthy people would likely be able to tolerate this interference, so it would not be life-threatening for them. However, the interference could seriously impact the health of people with lower levels of ACE2 or in people who need higher levels of ACE2 (perhaps because of high levels of the ACE protein, which produces Ang II, or because ACE2 products are beneficial for their pre-existing conditions). It might be even more dangerous for the latter because they would also have more binding sites for the virus. On the surface, this would explain the mortality distributions among pre-existing conditions, but it can also explain the age and sex demographics. Many of the pre-existing conditions develop or worsen with age, which explains the age-related trend in mortalities. Also, as noted earlier, data suggests that men, at least in China, are more likely to have these pre-existing conditions because of factors like smoking habits. Furthermore, the strength of the correlations between different medical conditions and ACE2 activity may also explain the trends in the order of the pre-existing conditions in mortality rates, although there is not enough data to be certain. For example, if diabetes and cardiovascular disease correlated more strongly with abnormal ACE2 levels than did chronic respiratory disease, that could explain its place on the list. Finally, since ACE2 is highly involved in maintaining vascular function, ACE2 inhibition may help explain the cardiovascular complications that have been observed recently in COVID-19. While existing data are not conclusive, they do warrant further research into this area. If the connection is proven, ACE2 levels could be used as identifiers for people at higher risk of death from COVID-19 and provide vital information for therapeutic development. Monitoring changes in ACE2 activity during infections in a variety of cases with different severities would provide additional data on this connection.

\section{References}

1. Tikellis C, Thomas MC (2012) Angiotensin-Converting Enzyme 2 (ACE2) Is a Key Modulator of the Renin Angiotensin System in Health and Disease. International Journal of Peptides.

2. Xu J, Fan J, Wu F, Huang Q, Guo M, et al. (2017) The ACE2/ Angiotensin-(1-7)/Mas Receptor Axis: Pleiotropic Roles in Cancer. Frontiers in Physiology 8: 276.

3. Hoffmann M, Kleine Weber H, Schroeder S, Krüger N, Herrler T, et al. (2020) SARS-CoV-2 Cell Entry Depends on ACE2 and TMPRSS2 and Is Blocked by a Clinically Proven Protease Inhibitor. Cell 181(2): 271-280.

4. Vital Surveillances (2020) The Epidemiological Characteristics of an Outbreak of 2019 Novel Coronavirus Diseases (COVID-19) in China. China CDC Weekly 41(2): 145-151.

5. Brit Long, William J Brady, Alex Koyfman, Michael Gottlieb (2020) Cardiovascular complications in COVID-19. The American Journal of Emergency Medicine.

6. Mesecar AD, Ratia K (2008) Viral destruction of cell surface receptors. Proceedings of the National Academy of Sciences 105(26): 8807-8808.

7. Flint SJ, Enquist LW, Racaniello VR, Skalka AM (2009) Principles of Virology (Third, Vol. 1). ASM Press. 